PROCEEDINGS OF THE

AMERICAN MATHEMATICAL SOCIETY

Volume 131, Number 4, Pages 1007-1010

S 0002-9939(02)06666-2

Article electronically published on September 25, 2002

\title{
INVERSE LIMITS OF ALGEBRAS AS RETRACTS OF THEIR DIRECT PRODUCTS
}

\author{
A. LARADJI
}

(Communicated by Carl G. Jockusch, Jr.)

\begin{abstract}
Inverse limits of modules and, more generally, of universal algebras, are not always pure in corresponding direct products. In this note we show that when certain set-theoretic properties are imposed, they even become direct summands.
\end{abstract}

Given a direct system $\left\{M_{i}\right\}_{i \in I}$ of modules, it is well known that $\lim _{i} M_{i}$ is a pure quotient of the direct sum $\bigoplus_{i \in I} M_{i}$. In contrast, the dual statement that inverse limits are pure submodules of corresponding direct products is not always true: For each prime number $p$, we can construct a descending chain $\left\{A_{n}\right\}_{n \in \mathbb{N}}$ of divisible abelian groups whose intersection $A$ is isomorphic to $\mathbb{Z} / p \mathbb{Z}$ (see [2, Exercise 6, p. 101]). Since divisibility is inherited by pure subgroups and direct products and since $A$ is not divisible, it follows that the inverse limit $A$ of the divisible groups $A_{n}$ is not pure in $\prod_{n \in \mathbb{N}} A_{n}$. However, as we shall show in this note, when certain set-theoretic conditions are imposed on an inverse system of modules, the inverse limit is a direct summand of the corresponding direct product. This is motivated by the following observation: Let $p$ be a prime number and let $J_{p}$ be the $p$-adic group $\lim \mathbb{Z} / p^{n} \mathbb{Z}$. As each $\mathbb{Z} / p^{n} \mathbb{Z}$ is finite, $J_{p}$ is linearly, and hence algebraically compact. (See [1] and 2].) Since, as can easily be proved, $J_{p}$ is pure in $\prod_{n} \mathbb{Z} / p^{n} \mathbb{Z}$, it follows that the canonical monomorphism $0 \rightarrow \lim \mathbb{Z} / p^{n} \mathbb{Z} \rightarrow \prod_{n} \mathbb{Z} / p^{n} \mathbb{Z}$ splits.

The purpose of this note is to generalize this result in both set-theoretic and universal algebraic directions. We refer to [4] and 3] for the various undefined notions used here from the theory of large cardinals and universal algebra, respectively. Recall that a tree is a poset $(T,<)$ such that for each $t \in T$ the set $\{s \in T: s<t\}$ of the predecessors of $t$ is well ordered by $<$. A subalgebra $B$ of an algebra $A$ is a retract of $A$ if there exists a homomorphism $g: A \rightarrow B$ whose restriction to $B$ is the identity on $B$; such a $g$ is called a retraction. A directed set $\{I ; \leq\}$ is $\lambda$-directed for some infinite cardinal $\lambda$, if every subset of $I$ of size less than $\lambda$ has an upper bound in $I$.

First, we need

Lemma 1. A subalgebra $B$ of an algebra $A$ is a retract of $A$ if and only if every system of equations over $B$ and with a solution in $A$ has a solution in $B$.

Proof. (Cf. 2, Proposition 22.3].) Suppose $B$ is a retract of $A$, with retraction $g$, and let $\Sigma$ be a system of equations over $B$ with a set of unknowns $\left\{x_{s}\right\}_{s \in S}$. If

Received by the editors April 3, 2001 and, in revised form, October 26, 2001.

2000 Mathematics Subject Classification. Primary 08B25, 03E55.

(C)2002 American Mathematical Society 
$\left\{a_{s}\right\}_{s \in S}$ is a solution of $A$ in $\Sigma$, then, clearly, $\left\{g\left(a_{s}\right)\right\}_{s \in S}$ is a solution of $\Sigma$ in $B$. Conversely, let $\Sigma$ be the system over $B$

$$
\begin{aligned}
x_{f\left(\left(a_{i}\right)_{i \in r(f)}\right)} & =f\left(\left(x_{a_{i}}\right)_{i \in r(f)}\right), \\
x_{b} & =b
\end{aligned}
$$

for any $a_{i} \in A, b \in B$ and any operation $f$ on $A$ (with arity $r(f)$ ), and where the unknowns are indexed by $A$. This system is solvable in $A$ by $x_{a}=a(a \in A)$. Thus, if $x_{a}=g(a)(a \in A)$ is a solution of $\Sigma$ in $B$, then the mapping $g: A \rightarrow B$ is a retraction.

Proposition 2. Let $\alpha$ be a limit ordinal, $\kappa$ be an infinite cardinal and $\left\{A_{i} ; \sigma_{i}^{j}\right\}_{i \leq j<\alpha}$ be a well-ordered inverse system of algebras with $\left|\sigma_{i}^{i+1}\left(A_{i+1}\right)\right|<\kappa<\operatorname{cf}(\alpha)$. Then the inverse limit $\lim _{\longleftarrow} A_{i}$ is a retract of $\prod_{i<\alpha} A_{i}$.

Proof. We first show that every system of equations over $\lim A_{i}$ and solvable in $\prod_{i<\alpha} A_{i}$ has a solution in $\lim _{\longleftarrow} A_{i}$. Let $\Sigma$ be a system of equations over $\lim _{\longleftarrow} A_{i}$ with unknowns $\left\{x_{s}\right\}_{s \in S}$, let $C$ be the set of all constants appearing in $\Sigma$, and suppose $\Sigma$ is solvable in $\prod_{i<\alpha} A_{i}$ by $\left\{a_{s}\right\}_{s \in S}$, say. For each $i<\alpha$, let $\Sigma^{i}$ be the system obtained from $\Sigma$ by replacing each $c$ in $C$ by its $i$-th coordinate $c(i)$ in $A_{i}$. Fix $s$ in $S$ and let $T_{s}^{i}=\left\{\sigma_{i}^{j}\left(a_{s}(j)\right): i \leq j<\alpha\right\}$, where $a_{s}(j)$ is the $j$-th coordinate of $a_{s}$. Partial-order $T_{s}=\bigcup_{i<\alpha} T_{s}^{i}$ by setting $x<y$ when $x \in T_{s}^{i}, y \in T_{s}^{j}$ and $\sigma_{i}^{j}(y)=x$ for some $i<j<\alpha$. It is easy to see that $\left(T_{s},<\right)$ is a tree of height $\alpha$. For any $x=\sigma_{i}^{j}\left(a_{s}(j)\right)$ in $T_{s}^{i}$, we have $x=a_{s}(i)$ or $x=\sigma_{i}^{i+1} \sigma_{i+1}^{j}\left(a_{s}(j)\right) \in \sigma_{i}^{i+1}\left(A_{i+1}\right)$, so that $T_{s}^{i} \subseteq\left\{a_{s}(i)\right\} \cup \sigma_{i}^{i+1}\left(A_{i+1}\right)$, and therefore $\left|T_{s}^{i}\right|<\kappa$. As $\alpha$ is a limit ordinal, $T_{s}$ has a branch $\left(\mu_{s}(i)\right)_{i<\alpha}$, by [5, Proposition 2.32, p. 304]. Since $\sigma_{i}^{j}\left(\mu_{s}(j)\right)=\mu_{s}(i)$ for all $i \leq j<\alpha$, we obtain that $\left(\mu_{s}(i)\right)_{i<\alpha} \in \lim A_{i}$ for each $s \in S$. Now we have $c(i)=\sigma_{i}^{j}(c(j))$ for all $i \leq j$, since $C \subseteq \varliminf_{\lim } A_{i}$, so that for all $j \geq i,\left\{\sigma_{i}^{j}\left(a_{s}(j)\right)\right\}_{s \in S}$ is a solution of $\Sigma^{i}$. By definition of $T_{s}$, for each $i<\alpha, \mu_{s}(i)=\sigma_{i}^{j}\left(a_{s}(j)\right)$ for some $j \geq i$, i.e. $\left\{\mu_{s}(i)\right\}_{s \in S}$ is a solution of $\Sigma^{i}$. Since $\left(\mu_{s}(i)\right)_{i<\alpha} \in \lim A_{i}$ for all $s$ in $S$, we infer that $\Sigma$ is solvable in $\lim A_{i}$, as required. It now follows that $\lim A_{i}$ is not empty (choose $\Sigma$ with $C=\varnothing$ ) and therefore is a subalgebra of $\prod_{i<\alpha} \overleftarrow{A_{i}}$, and that it is a retract of $\prod_{i<\alpha} A_{i}$, by Lemma 1 .

We next turn our attention to cardinals with the tree property stating that the cardinal satisfies König's Lemma. Recall that $\aleph_{0}$ and weakly compact (e.g. measurable) cardinals have the tree property, whereas $\aleph_{1}$ and singular cardinals do not.

Proposition 3. Let $\alpha$ be a limit ordinal, $\kappa$ be an infinite cardinal with the tree property, and $\left\{A_{i} ; \sigma_{i}^{j}\right\}_{i \leq j<\alpha}$ be a well-ordered inverse system of algebras with $\left|\sigma_{i}^{i+1}\left(A_{i+1}\right)\right|<\kappa \leq \operatorname{cf}(\alpha)$. Then $\lim _{A_{i}}$ is a retract of $\prod_{i<\alpha} A_{i}$.

Proof. If $\kappa<\operatorname{cf}(\alpha)$, use Proposition 22 Suppose that $\kappa=\operatorname{cf}(\alpha)$ with $\alpha=\sum_{t<\kappa} \alpha_{t}$, where $\alpha_{t}<\alpha$. Then, using the tree property of $\kappa$ and an argument similar to that of Proposition 2, we obtain that $\lim _{4} A_{i}$ is a subalgebra of $\prod_{i<\alpha} A_{i}$, and that $\lim _{A_{\alpha_{t}}} A_{\alpha_{t}}$, the inverse limit of the inverse family $\left\{A_{\alpha_{t}} ; \sigma_{\alpha_{t}}^{\alpha_{s}}\right\}_{t \leq s<\kappa}$, is a retract of $\prod_{t<\kappa} \overleftarrow{A_{\alpha_{t}}}$. Let $\varphi: \prod_{i<\alpha} A_{i} \rightarrow \prod_{t<\kappa} A_{\alpha_{t}}$ be the canonical projection. Then (see for example the proof of [3, Lemma 7, p. 133]), the restriction $\psi$ of $\varphi$ to $\lim A_{i}$ is an isomorphism $\lim A_{i} \rightarrow \lim A_{\alpha_{t}}$ and we have $\varphi f=g \psi$, where $f: \lim _{i} \rightarrow \prod_{i<\alpha} A_{i}$ and $g:$

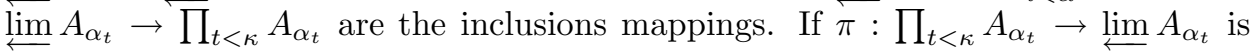


such that $\pi g$ is the identity, then $\psi^{-1} \pi \varphi f=\psi^{-1} \pi g \psi$ is the identity mapping on $\underset{\lim }{\longleftarrow} A_{i}$, and so $\lim _{\longleftarrow} A_{i}$ is a retract of $\prod_{i<\alpha} A_{i}$.

The conclusion of Proposition 3 can be arrived at for a wider class of inverse systems, provided $\kappa$ is a compact cardinal. An infinite cardinal $\lambda$ is compact if, for any set $S$, every $\lambda$-complete filter on $S$ can be extended to a $\lambda$-complete ultrafilter. Thus $\aleph_{0}$ is compact, and it is known that uncountable compact cardinals are necessarily measurable. We have

Proposition 4. Let $\left\{A_{i} ; \sigma_{i}^{j}\right\}_{i \in I}$ be an inverse system of algebras and let $\kappa$ be a compact cardinal such that $\{I ; \leq\}$ is $\kappa$-directed and $\left|\bigcup_{j>i} \sigma_{i}^{j}\left(A_{j}\right)\right|<\kappa$, for every $i \in I$. Then $\lim _{\longleftarrow} A_{i}$ is a retract of $\prod_{i \in I} A_{i}$.

Proof. We first show that $\lim _{\longleftarrow} A_{i}$ is non-empty. For each $i \in I$, let $p_{i} \in A_{i}, \pi_{i}$ : $\prod_{j \in I} A_{j} \rightarrow A_{i}$ be the $i$-th canonical projection, and let $T_{i}=\left\{\sigma_{i}^{j}\left(p_{j}\right): i, j \in I, i \leq\right.$ $j\}$. For every $J \in[I]^{<\kappa}=\{S \subseteq I:|S|<\kappa\}$, let $X_{J}=\left\{x \in \prod_{i \in I} T_{i}: \sigma_{i}^{j}\left(p_{j}\right)=p_{i}\right.$, for all $i, j \in J$ and $i \leq j\}$. Since $I$ is $\kappa$-directed and $\kappa$ is regular (compact cardinals

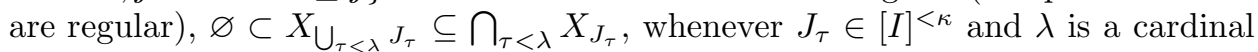
less than $\kappa$. It follows that the set $\left\{X_{J}\right\}_{J \in[I]<\kappa}$ generates on $\prod_{i \in I} T_{i}$ a $\kappa$-complete proper filter, which, as $\kappa$ is compact, can be extended to a $\kappa$-complete ultrafilter $U$. For each $Y \in U$, let $Y_{i}=\left\{x_{i} \in T_{i}: x=\left(x_{i}\right)_{i \in I} \in Y\right\}$ and let $U_{i}=\left\{Y_{i}: Y \in\right.$ $U\}$. As in the proof of [3, Theorem 1, p. 132], we obtain that $U_{i}$ is a $\kappa$-complete ultrafilter on $T_{i}$. By hypothesis $\left|T_{i}\right|<\kappa$, so that $U_{i}$ is a principal generated by a singleton $\left\{y_{i}\right\}$, say. Now, for all $i, j \in I, \pi_{i}^{-1}\left(\left\{y_{i}\right\}\right), \pi_{j}^{-1}\left(\left\{y_{j}\right\}\right)$ and $X_{\{i, j\}}$ are in $U$, so that $\pi_{i}^{-1}\left(\left\{y_{i}\right\}\right) \cap \pi_{j}^{-1}\left(\left\{y_{j}\right\}\right) \cap X_{\{i, j\}} \in U$. Therefore, if $i \leq j$, there exists $x=\left(x_{i}\right)_{i \in I} \in X_{\{i, j\}}$ such that $\sigma_{i}^{j}\left(y_{j}\right)=\sigma_{i}^{j}\left(x_{j}\right)=x_{i}=y_{i}$. This proves that $\longleftarrow A_{i}$ is non-empty, and thus a subalgebra of $\prod_{i \in I} A_{i}$. Next, let $\Sigma$ be a system of equations over $\lim _{\longleftarrow} A_{i}$ with unknowns $\left\{x_{s}\right\}_{s \in S}$ and constants $\{c\}_{c \in C}$, and suppose it is solvable in $\overleftarrow{\prod}_{i \in I} A_{i}$ by $\left\{a_{s}\right\}_{s \in S}$. For each $i \in I$, let $\Sigma^{i}$ be the system obtained from $\Sigma$ by replacing each $c$ in $C$ by its $i$-th coordinate $c(i)$ in $A_{i}$. Fix $s$ in $S$, and set $B_{i}^{s}=\left\{\sigma_{i}^{j}\left(a_{s}(j)\right): j \in I, i \leq j\right\}$. It is easy to see that $\left\{B_{i}^{s}\right\}_{i \in I}$ can be regarded as an inverse system of non-empty sets with bonding maps $\sigma_{i}^{j}(i \leq j)$. By the first part of this proof, $\lim _{\longleftarrow} B_{i}^{s}$ is non-empty. Clearly, if $\mu_{s} \in \lim _{\longleftarrow} B_{i}^{s}$, then $\left\{\mu_{s}\right\}_{s \in S}$ is a solution of $\Sigma$ in $\lim _{\longleftarrow}$. Now use Lemma 1

Remarks. The first part of the foregoing proof (that $\lim _{i} A_{i}$ is non-empty) is a straightforward adaptation of an argument of Grätzer [3, Theorem 1, p. 132], where he used ultrafilters to prove the classical theorem that inverse limits of finite nonempty sets are non-empty. Indeed, since $\aleph_{0}$ is compact, Proposition 4 generalizes both [3, Theorem 1, p. 132] (and hence König's Graph Lemma) and the observation on $J_{p}$ mentioned above. As was pointed out by the referee, Proposition 2 and its proof provide an alternative proof of [3, Theorem 1, p. 132] for inverse systems $\left\{A_{i} ; \sigma_{i}^{j}\right\}_{i<\alpha}$ : If $\operatorname{cf}(\alpha)=\omega$, then, for some countable cofinal subset $\left\{i_{n}: n \in \mathbb{N}\right\}$ of $\alpha, \lim A_{i}$ is isomorphic to $\lim _{i_{n}}$ which, using the proof of Proposition 2 with König's Lemma, is non-empty. If $\operatorname{cf}(\alpha)>\omega$, then the same follows again by using $\kappa=\omega$ in Proposition 2 ,

Corollary 5. Let $\kappa$ be a compact cardinal and let $\left\{A_{i}\right\}_{i \in I}$ be an inverse system of algebras such that $\{I ; \leq\}$ is $\kappa$-directed and $\left|A_{i}\right|<\kappa$ for all $i \in I$. Then $\lim _{i} A_{i}$ is a retract of $\prod_{i \in I} A_{i}$. 


\section{ACKNOWLEDGMENTS}

The author gratefully acknowledges the support of King Fahd University of Petroleum and Minerals. He also thanks the referee for comments that led to several improvements.

\section{REFERENCES}

1. L. Fuchs, Note on linearly compact abelian groups, J. Austral. Math. Soc. 9 (1969), 433-440. MR 39:6979

2. L. Fuchs, Infinite Abelian Groups I, Academic Press, New York, 1970. MR 41:333

3. G. Grätzer, Universal Algebra, Second Edition, Springer-Verlag, New York, 1979. MR 80g:08001

4. T. Jech, Set Theory, Academic Press, New York, 1978. MR 80a:03062

5. A. Levy, Basic Set Theory, Springer-Verlag, Berlin, 1979. MR 80k:04001

Department of Mathematical Sciences, King Fahd University of Petroleum and Minerals, Dhahran 31261, Saudi Arabia

E-mail address: alaradji@kfupm.edu.sa 